\title{
Toward Interactive User Guiding Vessel Axis Extraction from Gray-scale Angiograms: An Optimization Framework ${ }^{\star}$
}

\author{
Wilbur C.K. Wong and Albert C.S. Chung \\ Lo Kwee-Seong Medical Image Analysis Laboratory, Department of Computer \\ Science and Engineering, The Hong Kong University of Science and Technology \\ \{cswilbur, achung\}@cse.ust.hk
}

\begin{abstract}
We propose a novel trace-based method to extract vessel axes from gray-scale angiograms without preliminary segmentations. Our method traces the axes on an optimization framework with the bounded spherical projection images and the sum of squared difference metric. It does not take alternate steps to search the next axial point and its tangent as in other trace-based algorithms, instead the novel method finds the solution simultaneously. This helps avoid U-turns of the trace and large spatial discontinuity of the axial points. Another advantage of the method is that it enables interactive user guidance to produce continuous tracing through regions that contain furcations, disease portions, kissing vessels (vessels in close proximity to each other) and thin vessels, which pose difficulties for the other algorithms and make re-initialization inevitable as illustrated on synthetic and clinical data sets.
\end{abstract}

\section{Introduction}

Segmentation of 3-D angiograms can provide patient-specific 3-D vascular models for diagnosis and prognosis. Nevertheless, if one wants to: (a) study quantitatively the vessel attributes over a particular vessel population [1]; (b) generate fly-throughs in virtual endoscopy [2]; or (c) perform real-time registration of 3-D vessels to X-ray angiograms [3], then extraction of the vessel axis is required. This usually demands further post-processing on the vascular segmentation. Topological thinning [4], voxel encoding technique [5, 2, and Voronoi diagram 6] had been investigated for the construction of vessel axes from the segmentation. Hybrid approaches were also proposed [7,8]. In order to obtain a satisfactory vessel axis extraction with the above algorithms, a topologically and morphologically correct vascular segmentation (i.e., with no holes and cavities) is necessary, which in fact may be difficult to obtain from clinical data sets.

Skeleton-based vascular segmentation algorithms $9,10,11,12$, provide an alternative mean to kill two birds with one stone. In those algorithms, vessel axes are extracted and the local vessel widths are estimated in the meantime, and

\footnotetext{
^ This work is supported by the HK Research Grants Council under grant 612305.
} 


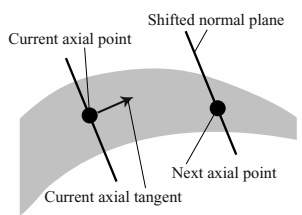

(a)

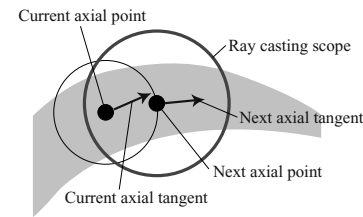

(b)

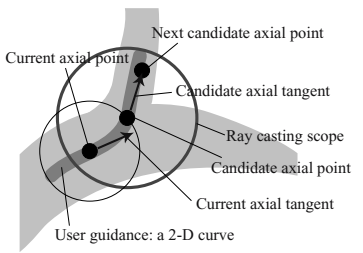

(c)

Fig. 1. Illustrations of (a) a typical trace-based method, (b) the optimization framework for axis tracing and (c) tracing with user guidance on the optimization framework

therefore a vascular segmentation can be obtained as a by-product. Technique that produces vessel axes without segmentation was also proposed [13]. Generally, those axis extraction methods [9, 11, 10, 12 consist of two core alternate tasks, the generation of the axial tangent and the computation of the next axial point. A trace is initiated by a manual seed point. The axial tangent of the seed is then generated. This tangent helps define a cross-sectional plane (normal plane), which is used to compute the next axial point after the plane is shifted forward along the tangent direction, as illustrated in Fig. 1(a). This process keeps iterating until a termination criterion is met or the user pre-empts the tracing.

Nevertheless, the trace obtained with the aforementioned methods may go astray and make U-turns in regions that contain furcations, disease portions and kissing vessels [11. Therefore, re-initialization of the trace at the proximal end of the side branch, and the immediate disease-free and kissing-free portions is inevitable. As a result, a disconnected vessel axis may be produced.

To alleviate this problem, we propose a novel trace-based approach to extracting a continuously connected vessel axis, in which we re-formulate the axis tracing problem on an optimization framework. The objective function is built upon two bounded spherical projection images and the sum of squared difference metric. Such framework allows seamless integration of interactive user guidance (2-D curves drawn on the screen by the user) on the axis extraction so as to drive the trace along the user-defined paths to fly through those problematic regions. Our method does not take alternate steps to search the axial points and tangents as suggested in [9, 11, 10, 12. Indeed it finds the next axial point and its tangent simultaneously and guarantees that the axial points are distanced from the preceding and the succeeding ones by a user-defined distance, which effectively helps avoid U-turns of the trace and large spatial discontinuity of the axial points. Experimental results on synthetic and clinical data sets show that our method can extract continuous and less jittering vessel axis in the regions that contain furcations, kissing vessels and thin vessels (in diameter down to 2 voxels). Furthermore, with the interactive user guidance, it can produce continuous traces to side branches at furcations and bypass disease portions. 


\section{Optimization Framework for Axis Tracing}

\subsection{Objective Function}

The objective function to be optimized is based upon a bounded spherical projection (BSP) image generated at a candidate axial point with reference to its associated tangent. The BSP image is created in a similar fashion as the spherical MIP image described in [14, except the following: (1) our projection is bounded, i.e., the spherically casted rays are not extended infinitely, (2) the pixel intensities of the BSP image are the normalized cumulative sums of the voxels along the casted rays and (3) the polar coordinate is originated from the direction of the associated tangent. Figs. 2(a)-(d) show some of the BSP images generated on an image volume with a solid straight tube (intensities inside are higher than the outside) at different 3 -D points and their associated tangents as depicted in Figs. 2(e)-(h). It is observed that there is a special pattern (three horizontal strips) on the BSP image if the length of the projection bound $(r)$ is greater than the tube radius (we set $r$ equal to $1.5 \times$ the tube radius), the 3 -D point is on the desire axis and its associated tangent is aligned with the axis orientation, see Figs. 2(a) and2(e). As such, through measuring the similarity between this BSP image $(\hat{\mathcal{I}})$ and those generated at the candidate axial points with the associated axial tangents, we can find the next axial point and the associated axial tangent simultaneously by the following minimization:

$$
\boldsymbol{p}^{*}=\arg \min _{\boldsymbol{p}}\left(\omega_{g} \cdot \omega_{s} \cdot S S D(\mathcal{I}(\boldsymbol{p}), \hat{\mathcal{I}})\right)
$$

where $\boldsymbol{p}$ is a parameter vector that contains the candidate axial point, its associated tangent and the length $r, \boldsymbol{p}^{*}$ denotes the solution of the minimization, $\omega_{g}$ and $\omega_{s}$ are the multiplicative weights for the integration of user guidance and the constraint on axis smoothness (see Sec. 2.3 for details), the function $S S D$ returns the sum of squared difference (SSD) metric of the two arguments, $\mathcal{I}(\boldsymbol{p})$ is the BSP image generated with the given parameter vector.

\subsection{Parameter Vector}

As depicted in Fig. 1(b) (which shows a 2-D version for better illustration), the location of the next axial point $\boldsymbol{x}_{i+1}$ and its associated tangent $\hat{t}_{i+1}$ can be expressed as spherical coordinates, $\left(\theta_{x}^{i+1}, \phi_{x}^{i+1}\right)$ and $\left(\theta_{t}^{i+1}, \phi_{t}^{i+1}\right)$, relative to the current axial points $\boldsymbol{x}_{i}$, respectively. The coordinates $\phi_{x}^{i+1}$ and $\phi_{t}^{i+1} \in[0, \pi]$ denote the polar angles originated from $\hat{t}_{i}$ and $\left(\boldsymbol{x}_{i+1}-\boldsymbol{x}_{i}\right)$, respectively, $\theta_{x}^{i+1}$ and $\theta_{t}^{i+1} \in[0,2 \pi)$ denote the azimuthal angles on the normal planes. Mathematically,

$$
\boldsymbol{x}_{i+1}=\boldsymbol{x}_{i}+\mathbf{R}_{t}^{i}\left[\begin{array}{c}
d \cos \theta_{x}^{i+1} \sin \phi_{x}^{i+1} \\
d \sin \theta_{x}^{i+1} \sin \phi_{x}^{i+1} \\
d \cos \phi_{x}^{i+1}
\end{array}\right] \quad \text { and } \quad \hat{t}_{i+1}=\mathbf{R}_{x}^{i}\left[\begin{array}{c}
\cos \theta_{t}^{i+1} \sin \phi_{t}^{i+1} \\
\sin \theta_{t}^{i+1} \sin \phi_{t}^{i+1} \\
\cos \phi_{t}^{i+1}
\end{array}\right]
$$

where $d=1$ voxel is a user-defined step size of the trace, $\mathbf{R}_{t}^{i}$ and $\mathbf{R}_{x}^{i}$ are the 3-D rotation matrices defined by using $\hat{t}_{i}$ and the unit vector of $\left(\boldsymbol{x}_{i+1}-\boldsymbol{x}_{i}\right)$ as the Zaxis, respectively, their normal vectors as $\mathrm{Y}$-axis and binormal vectors as $\mathrm{X}$-axis. 


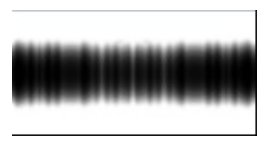

(a)

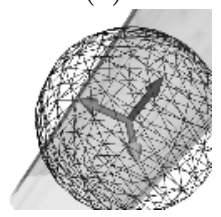

(e)

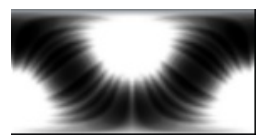

(b)

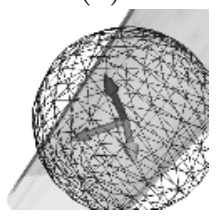

(f)

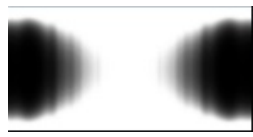

(c)

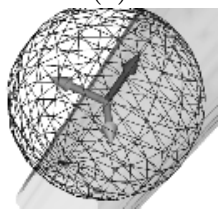

(g)

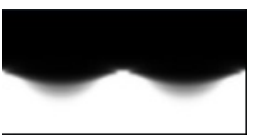

(d)

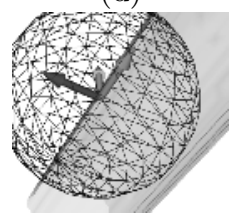

(h)

Fig. 2. (a)-(d) The BSP images (azimuthal and polar angles are enumerated in the Xand $\mathrm{Y}$-axis, respectively) generated on an image volume with a solid straight tube (the surfaces are for visualization only) at different 3-D points and their associated tangents (the dark gray arrows) as depicted in (e)-(h). The wireframe-spheres define the scope of the bounded spherical projection.

Including the length of the projection bound $r_{i+1}$ of the corresponding candidate BSP image, we have a 5 -D parameter vector, $\boldsymbol{p}_{i+1}=\left[\theta_{x}^{i+1}, \phi_{x}^{i+1}, \theta_{t}^{i+1}, \phi_{t}^{i+1}, r_{i+1}\right]$. In order to avoid backward tracing, we set the search domain of the polar angles to $[0, \pi / 3]$. In addition, we force $r_{i+1} \in\left[(1-q) r_{i},(1+q) r_{i}\right]$, where $q=0.5$ controls the allowable length change in percentage of $r_{i}$ to prevent sudden changes in the length $r$ when the trace flies through bifurcations and disease portions.

\subsection{Integration of User Guidance and Axis Smoothness Constraint}

User guidance is expressed as a set of user-drawn 2-D curves on the screen. They help steer the vessel axis traced in 3-D by giving reference paths on which the perspective projected trace should follow on the screen. This idea is illustrated in Fig. 1(c). Suppose the trace follows the main vessel from left to right without any guidance. If the user wants to guide the trace towards the side branch, a 2-D curve should be drawn on the screen as given.

We implement such guidance as the multiplicative weight $\omega_{g}$ in the objective function (see Eqn. 11). The weight is defined as follows,

$$
\omega_{g}=\operatorname{Rescale}\left(\prod_{j=1}^{2}\left(1-\exp \left(-\frac{s_{j}^{2}}{2 \sigma_{g}^{2}}\right)\right), \omega_{g}^{\min }, \omega_{g}^{\max }\right)
$$

where the function Rescale linearly transforms the first argument from $[0,1]$ to $\left[\omega_{g}^{\min }, \omega_{g}^{\max }\right]$, we set $\omega_{g}^{\min }=0.1$ and $\omega_{g}^{\max }=1$ which are the minimum and maximum values of $\omega_{g}, s_{1}$ and $s_{2}$ are the shortest distances from the perspective projected points of the next two succeeding candidate axial points $\boldsymbol{x}_{i+1}^{\prime}$ and $\boldsymbol{x}_{i+2}^{\prime}$ to the 2-D curves, respectively. $\boldsymbol{x}_{i+2}^{\prime}$ is estimated from the candidate axial tangent $\hat{t}_{i+1}^{\prime}$ at $\boldsymbol{x}_{i+1}^{\prime}$ as follows: $\boldsymbol{x}_{i+2}^{\prime}=\boldsymbol{x}_{i+1}^{\prime}+d \hat{t}_{i+1}^{\prime}$. The variable $\sigma_{g}=3$ screen voxels controls the guiding influence over the candidate axial points. A smaller weight 
is obtained if $\boldsymbol{x}_{i+1}^{\prime}$ and $\boldsymbol{x}_{i+2}^{\prime}$ are closer to the curve on the screen. User guidance with more than one $2-\mathrm{D}$ curve is demonstrated in Section 3 .

Axis smoothness is constrained in the same fashion. The multiplicative weight $\omega_{s}$ in Eqn. 1 that favors smooth vessel axis is defined as follows,

$$
\omega_{s}=\operatorname{Rescale}\left(1-\exp \left(-\frac{(1-|\overline{\hat{t}} \cdot \hat{v}|)^{2}}{2 \sigma_{s}^{2}}\right), \omega_{s}^{\min }, \omega_{s}^{\max }\right)
$$

where $\omega_{s}^{\min }=0.7$ and $\omega_{s}^{\max }=1$ are the minimum and maximum values of the weight, $\overline{\hat{t}}$ is the mean vector of $\hat{t}_{i}$ and $\hat{t}_{i+1}^{\prime}, \hat{v}$ is the normalized vector of $\left(\boldsymbol{x}_{i+1}^{\prime}-\boldsymbol{x}_{i}\right)$ and $\sigma_{s}=0.3$ controls the degree of the axis smoothness (the smaller $\sigma_{s}$ is the smoother the axis).

\subsection{Initialization and Optimization}

The manually selected seed point may only be picked in close proximity to the vessel axis of interest. To initialize a trace, we need to find the first axial point $\boldsymbol{x}_{0}$ within the neighborhood of the seed point. This is done by locating the local maxima in a Gaussian smoothed version of the image volume. For the estimation of the initial projection bound length $r_{0}$ and the initial axial tangent $\hat{t}_{0}$, we perform the analysis of the Hessian matrix and compute the ridge direction (defined by $\hat{t}$ ) at each scale (defined by $r$ ) in a discrete scalespace [10]. Then we set the values of $r_{0}$ and $\hat{t}_{0}$ to the pair $(r, \hat{t})$ that gives minimum $S S D\left(\mathcal{I}^{\prime}, \hat{\mathcal{I}}\right)$, where $\mathcal{I}^{\prime}$ is the BSP image generated at $\boldsymbol{x}_{0}$ with the pair $(r, \hat{t}) . \hat{t}_{0}$ is flipped if an opposite trace is desired. We apply a modified version of $(1+1)$-Evolution Strategy (ES) for the optimization (modification is referred to the optimization with simple bounds on the parameters). (1+1)-ES is a special type of the Evolutionary Algorithms with both the population size and the number of children generated equal to one, which has an automatic step size and provides search direction adaptation [15]. Thus it has the ability to step out of non-optimal minima and can provide the axis tracing problem with a long capture range.

\section{Results}

We have tested the proposed method on three synthetic data sets and a clinical data set (3-D rotational angiography) available at http://www . gris . uni-tuebingen . de/ areas/scivis/volren/datasets/datasets.html, courtesy of Philips Research, Hamburg, Germany. The design of the synthetic data took several challenging vascular structures for the trace-based axis extraction methods into consideration: kissing vessels, a bifurcation, and a typical saccular aneurysm in the cerebral circulation.

Figs. 33(a)-(c) and 4 show the experimental results obtained from the newly proposed method on the synthetic and clinical data sets, respectively. The black axes in those figures are traced without any user guidance. It is evident that the vessel axes extracted from the kissing vessels (see Figs. 3(a) and 4(a)), at 


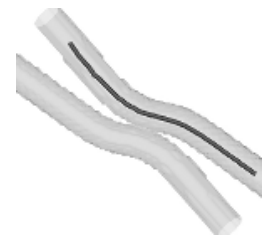

(a)

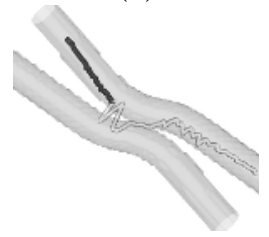

(e)

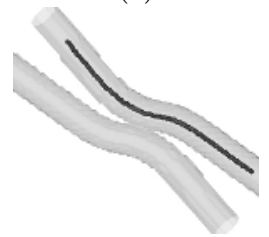

(i)

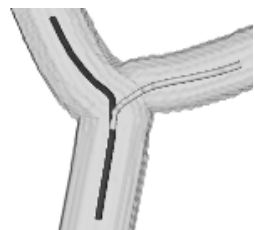

(b)

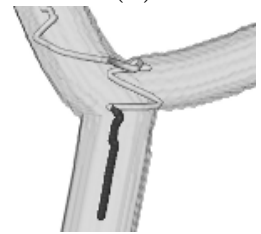

(f)

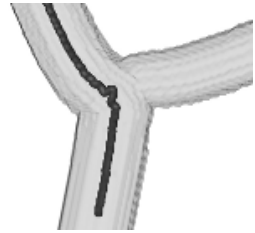

(j)

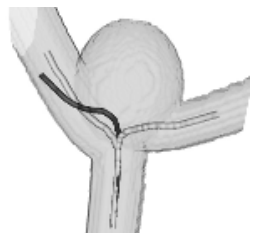

(c)

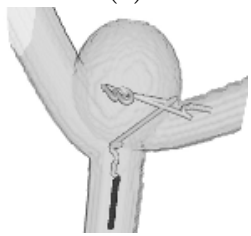

(g)

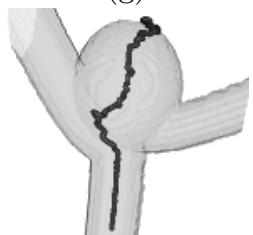

(k)

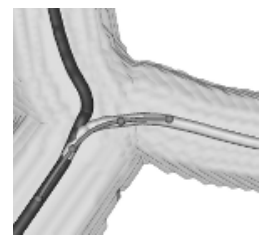

(d)

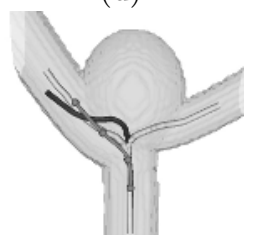

(h)

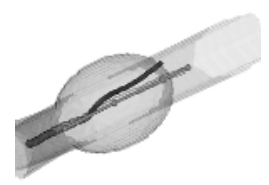

(1)

Fig. 3. Results obtained from (a)-(c) our novel method, the methods proposed by (e)(g) Aylward and Bullitt [10] and (i)-(k) Shim et al. [12] on the synthetic data sets which contain kissing vessels, a bifurcation and a saccular aneurysm. The 2-D curves drawn on the screen for user guidance on the tracing are given in (d), (h) and (l). The semi-transparent surfaces are for visualization only.

the furcations (see Figs. 3(b) and 4(b)), and from the thin vessel $(<2$ voxels in diameter, see Fig. 4 (c)) are satisfactory. Due to the existence of the saccular aneurysm (see Fig. 3(c)), the axis extracted are steered, as a result it is deviated from the desired vessel axis. User guidance is therefore needed to drive the trace towards the target axis in this case, as well as to the side branches of the bifurcations shown in Figs. 3(b)-(c). The user-drawn 2-D curves (dark gray in color with circles, those circles are the 2-D locations picked by the user to compose the curves) are depicted in Figs. 3(d), 3(h) and 3(l). The curve shown in Fig. 3(d) helps guide the trace (light gray in color) to go to the right side branch of the bifurcation given in Fig. 3(b). The other two curves given in Figs. 3(h) and 3(l) altogether steer the trace (in light gray) to the left side branch of the saccular aneurysm shown in Fig. 3(c). Two extra 2-D curves are needed to produce the trace to the right side branch, however, they are not shown due to page limitation.

As a comparison, we have also tested the methods presented in [10,12] on the data sets. Figs. $3(\mathrm{e})-(\mathrm{g})$ and $3(\mathrm{i})-(\mathrm{k})$ show the results obtained from Aylward and Bullitt's [10] and Shim et al. [12 methods on the synthetic data sets, respectively. The shorter black axes shown in Figs. 3(e)-(g) are the vessel axes extracted with the large spatial discontinuity detection (LSDD). Such 


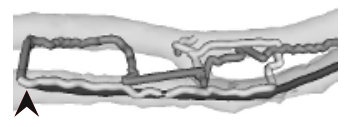

(a)

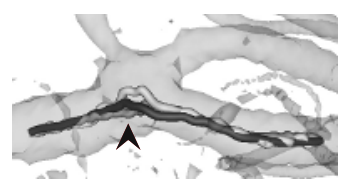

(b)

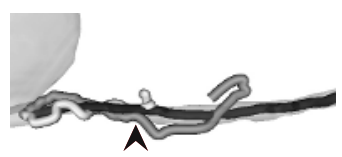

(c)

Fig. 4. Results on the clinical data set. The black, dark and light gray axes are the vessel axes obtained from ours, Aylward and Bullitt's [10] and Shim et al. 12 methods, respectively, on different vascular regions. The semi-transparent surfaces are for visualization only.

detection mechanism preempts the tracing if the next axial points is $>1$ voxel apart from the current one. It is observed that, with the detection, Aylward and Bullitt's method stops the tracing just before entering the kissing region, bifurcation and disease region. The method, however, produces axes (in gray) with a large axial point displacement in the aforementioned regions if no LSDD. Shim et al. method gives similar results except for the kissing vessels. In addition, several U-turns can be observed at the bifurcation and in the aneurysmal sac. Comparisons on the clinical data set are presented in Fig. 4, in which the dark and light gray axes are the vessel axes obtained from Aylward and Bullitt's, and Shim et al. methods, respectively. In the regions that contain kissing vessels, a furcation and a thin vessel, the axes extracted from the two methods is either jittered in between the two adjacent vessels, dragged towards the center of the furcation or off the target axis when the vessel is too thin to be traced. In general, the compared two methods produce vessel axis with more jitters than ours.

Furthermore, we have extracted the axes of nine major vessels, including the internal carotid artery (ICA), the two anterior cerebral arteries (ACA), a few branches of the middle cerebral artery (MCA) and the posterior communicating artery $(\mathrm{PCoA})$, in the clinical data set with our method. They are depicted in Fig. 5)(a). An MIP image is shown in Fig. 5(b) for reference. User guidance is given at the furcations to steer the traces towards the side branches and at the base of the aneurysm to bypass the disease portion (highlighted by arrows). It is demonstrated that our method can trace vessel segments, including the side branches and that in the disease region. Since the other algorithms do not support user guidance, they are not tested in this study. To study the repeatability of the method, the ICA is traced for five times. The axes extracted are shown in Fig. 5(c). Visually, there is no significant discrepancy amongst those axes.

We have also evaluated quantitatively the extracted vessel axes with the manually drawn axes built with reference to the reformatted cross-sectional images along the vessels. The mean Euclidean distance (ED) errors (maximum errors are given in the parentheses) in percentage of diagonal length of the voxel $(\Delta V)$ are listed in Table 1. The error is defined as the shortest ED from an axial point of the extracted axis to the manually drawn axis. Apparently Aylward and Bullitt's method with LSDD performs better according to the errors tabulated. However, it produces preempted traces upon large spatial discontinuity is encountered (the locations are highlighted by arrows in Figs. 4 and 5 (c)). Compared with 


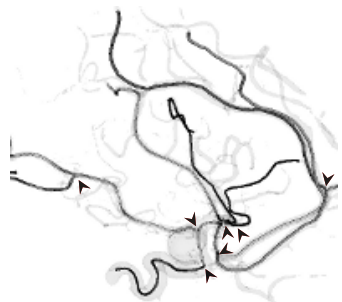

(a)

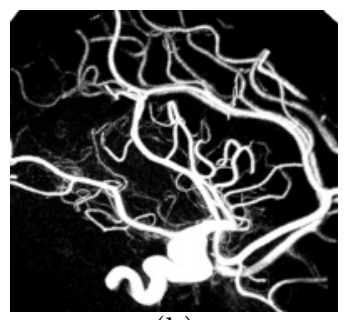

(b)

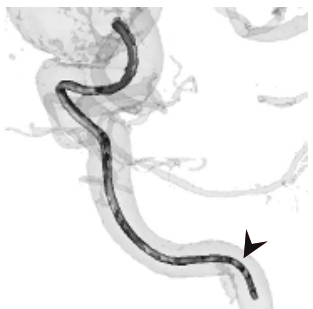

(c)

Fig. 5. (a) Extracted axes of nine major vessels in the clinical data set, including ICA, two ACA, a few branches of MCA and PCoA. (b) MIP image. (c) Axis of ICA extracted at five difference times. The semi-transparent surfaces are for visualization only.

Table 1. The Euclidean distance errors in percentage of diagonal length of the voxel between the extracted vessel axes and the manually drawn axes. The mean errors are tabulated with the maximum errors in the parentheses. The synthetic data sets are those shown in Fig. 3. The clinical data sets of sub-regions, major vessels and ICA are those presented in Figs. 4, 5(a) and 5(c), respectively.

\begin{tabular}{lcccc}
\hline & & Aylward and Bullitt & Aylward and & Shim et al. \\
Data Sets & Ours & with LSDD [10] & Bullitt [10] & [12] \\
\hline \hline Synthetic & $29 \%(126 \%)$ & $16 \%(140 \%)$ & $464 \%(1274 \%)$ & $304 \%(798 \%)$ \\
Clinical (Sub-regions) & $23 \%(60 \%)$ & $10 \%(53 \%)$ & $96 \%(182 \%)$ & $70 \%(228 \%)$ \\
Clinical (Major vessels) & $29 \%(100 \%)$ & - & - & - \\
Clinical (ICA) & $32 \%(77 \%)$ & $3 \%(76 \%)$ & $46 \%(137 \%)$ & $6 \%(86 \%)$ \\
\hline
\end{tabular}

the other algorithms which produce axes with large errors in the synthetic and several sub-regions of the clinical data sets, our method is capable of extracting the complete axes of the vessel segments consistently with mean errors $<\Delta V / 3$ and maximum errors $\approx \Delta V$. Concerning the processing time, the axes of the nine major vessels took $<15 \mathrm{~min}$ to extract with the novel method on a 2.0 $\mathrm{GHz}$ PC. The manual process, by contrast, took 169 min to finish.

\section{Discussion and Conclusion}

We have proposed a novel trace-based method which enables interactive user guiding of the trace to fly through challenging areas, e.g., vascular regions that contain furcations, disease portions, kissing vessels and thin vessels. The method traces a vessel axis on an optimization framework with the bounded spherical projection (BSP) images and the sum of squared difference (SSD) metric. Interactive user guidance (2-D curves that drawn on the screen by the user to steer the trace) and axis smoothness constraint are integrated seamlessly as the multiplicative weights in the objective function. Experimental results on synthetic and clinical data have shown that our method can extract continuous and less jittering vessel axes, as compared with the other two trace-based algorithms. 
Several potential extensions to the method are of interest for further research. For instance, reducing the user guidance on tracing the side branches of a furcation, extending the method to trace axes of anatomies other than blood vessels (especially those with asymmetric cross-sections) with different reference images/models and similarity measures (i.e. other than BSP images and SSD metric), and incorporating the skeleton-based segmentation algorithms so as to help producing topologically and morphologically correct vascular segmentations.

\section{References}

1. Bullitt, E., et al.: Analyzing attributes of vessel populations. MedIA 9 (2004) 39-49

2. Farag, A.A., et al.: Reliable fly-throughs of vascular trees. Technical report, Dept. Elec. Comput. Eng., Univ. of Louisville (2004)

3. Kita, Y., et al.: Real-time registration of 3D cerebral vessels to X-ray angiograms. In: MICCAI. (1998) 1125-1133

4. Palágyi, K., et al.: A sequential 3D thinning algorithm and its medical applications. In: IPMI. (2001) 409-415

5. Zhou, Y., Toga, A.W.: Efficient skeletonization of volumetric objects. TVCG 5 (1999) 196-209

6. Ogniewicz, R.L., Kubler, O.: Hierarchy Voronoi skeletons. PR 28 (1995) 343-359

7. Flasque, N., et al.: Acquisition, segmentation and tracking of the cerebral vascular tree on 3D magnetic resonance angiography images. MedIA 5 (2001) 173-183

8. Hassouna, M.S., et al.: Differential fly-throughs (DFT): a general framework for computing flight paths. In: MICCAI. (2005) 654-661

9. Hernández-Hoyos, M., et al.: A deformable vessel model with single point initialization for segmentation, quantification and visualization of blood vessels in 3D MRA. In: MICCAI. (2000) 735-745

10. Aylward, S., Bullitt, E.: Initialization, noise, singularities, and scale in height ridge traversal for tubular object centerline extraction. TMI 21 (2002) 61-75

11. Wink, O., et al.: Fast delineation and visualization of vessels in 3-D angiographic images. TMI 19 (2000) 337-346

12. Shim, H., et al.: Partition-based extraction of cerebral arteries from CT angiography with emphasis on adaptive tracking. In: IPMI. (2005) 357-368

13. Krissian, K., et al.: Fast and automatic vessel centerline detection for MRA. In: ISMRM. (2002)

14. van der Weide, R., et al.: CTA-based angle selection for diagnostic and interventional angiography of saccular intracranial aneurysms. TMI 17 (1998) 831-841

15. Styner, M., et al.: Parametric estimate of intensity inhomogeneities applied to MRI. TMI 19 (2000) 153-165 